\section{Usage of central venous catheter for delayed traumatic pneumothorax}

JIUN-HUNG GENG • WEI-CHE LEE • HSING-LIN LIN
JIUN-HUNG GENG $(\bowtie)$

Department of Urology Kaohsiung Medical University

Kaohsiung, Taiwan

100 Shih-Chuan 1st Road

Kaohsiung 807, Taiwan

Phone: (07) 3208212

Fax: (07) 3211033

E-mail: u9001090@hotmail.com

JIUN-HUNG GENG MD •

WEI-CHE LEE •

HSING-LIN LIN

Department of Surgery

WEI-CHE LEE •

HSING-LIN LIN

Division of Trauma

WEI-CHE LEE •

HSING-LIN LIN

Division of General Surgery Medicine

WEI-CHE LEE •

HSING-LIN LIN

Department of Emergency Medicine

JIUN-HUNG GENG MD

Department of Urology

Kaohsiung Medical University Hospital

\begin{abstract}
Pneumothorax, whether spontaneous, iatrogenic or traumatic, frequently requires drainage, especially in the face of positive pressure ventilation or general anesthesia. The traditional approach with large-bore, rigid chest tube is associated with significant pain and various complications. Recently, less invasive modalities such as pigtail catheter or fine-needle aspiration have been used in selected patients. We report a case of delay-onset pneumothorax after trauma and the patient was treated successfully with the easily available central venous catheter for drainage.
\end{abstract}

Key words: thoracic injury, delayed traumatic pneumothorax, central venous catheter, thoracotomy

\section{Introduction}

Traumatic pneumothorax is a cause of preventable death. It has been reported to be present in 2-55\% of patients suffering blunt thoracic trauma. (1-5) In recent studies, delayed traumatic pneumothorax has ranged from 2-5.42\% and patients sustaining blunt thoracic trauma with rib fractures should be admitted for close observation. $(6,7)$
Pneumothorax, whether spontaneous, iatrogenic or traumatic, frequently requires drainage, especially in the face of positive pressure ventilation or general anesthesia. The traditional approach, with a large-bore, rigid chest tube, is associated with significant pain and various complications. Recently, less invasive modalities such as a pigtail catheter or fine-needle aspiration have been used in selected patients. (8-11) However, their usefulness in the small number of occurrences of delayed traumatic pneumothorax has not been well established. To our knowledge, there has been no report about delayed traumatic pneumothorax treated by central venous catheter drainage.

Herein, we report a patient who needed surgical treatment for a delayed-onset, traumatic pneumothorax and who was treated safely and successfully with a central venous catheter.

\section{Case report}

A 48-year-old female was brought to our emergency department (ED) after having had a motorcycle traffic acci- 


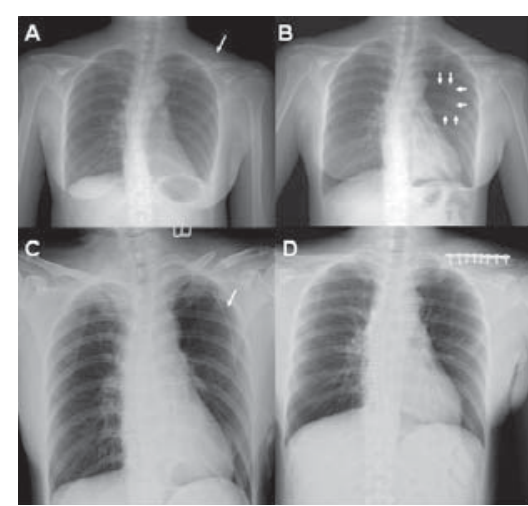

Figure 1 A. Left mid- clavicular fracture noted (arrow). No pneumothorax found. B. One day later, the chest $X$-ray revealed a left pneumothorax. Collapsed lung is marked by small arrow. C. After central venous catheter insertion (arrow), obvious lung expansion was noted. D. There was no recurrent pneumothorax and the drain was left for 7 days with little pain, no complications, and no catheter occlusion.

dent and thereby sustaining a left thoracic contusion injury. She had no head injury and no loss of consciousness at the scene. She was surveyed following the protocol of ATLS (advanced traumatic life support). On physical examination, vital signs were within normal range, and bilateral breath sounds were clear. There was tenderness over her left shoulder area and she had difficulty lifting her forearm. Neither obvious ecchymosis nor tenderness points over her anterior chest wall were noted. Chest radiography showed a left mid clavicular fracture, but no evidence of rib fractures, pneumothorax or hemothorax (figure 1A). A left shoulder sling was used and she was scheduled for open reduction with internal fixation (ORIF) of the clavicular fracture the following day. However, the X-ray taken on the following morning revealed a small amount of delayed pneumothorax (figure 1B). She did not have respiratory distress. Considering the self-limiting nature of the pneumothorax and the fact that she was about to undergo an operation under general anesthesia, a small caliber central venous catheter was used for drainage.

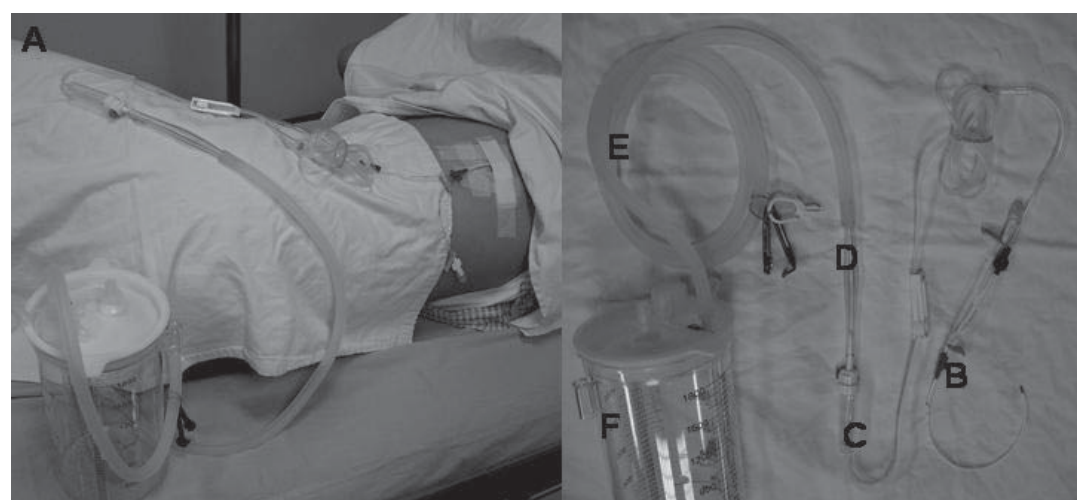

Figure 2 A. An 18-gauge, $20-\mathrm{cm}$ double lumen central venous catheter kit (no. 904219; SUNGWON MEDICAL Co., Korea) was used and the catheter was fixed to the skin at $17 \mathrm{~cm}$ from the tip. B. Central venous catheter used to enter the pleural space. C. Infusion set ("SIGMA" infusion set). D+E. "PAHSCO" surgical connecting tube and F. "PAHSCO" wound drainage collection bottle, Taiwan. The central venous catheter was connected tightly to the chest bottle via an infusion set.

An 18-gauge, 20-cm double lumen central venous catheter kit (no. 904219; SUNGWON MEDICAL Co., Korea) was used ( figure 2A). The patient was placed in the supine position and, under sufficient partial anesthesia with lidocaine, the 18-gauge catheter over the 20-gauge needle was entered into the pleural space along the anterior axillary line and fourth intercostal space. After the needle aspirated air, the Seldinger technique (12) was then employed to insert the line. A blunt guidewire was passed through the needle, and the needle was then removed. A dilating device was passed over the guidewire to slightly enlarge the tract, and the central line itself was then passed over the guidewire, which was then removed. The catheter was fixed to the skin at $17 \mathrm{~cm}$ from the tip and was connected tightly to the chest bottle ("PAHSCO" surgical connecting tube and "PAHSCO" wound drainage collection bottle, Taiwan) via an infusion set ("SIGMA" infusion set) (figure 2B-F).

After the central venous catheter was inserted, a negative pressure by $-10 \mathrm{~cm}$ $\mathrm{H}_{2} \mathrm{O}$ suction was applied shortly and lung expansion was noted in the followup chest X-ray (figure 1C). Then, the orthopedic surgeon performed the ORIF procedure smoothly. The drain was left for 7 days without obvious pain, complications, nor catheter occlusion (figure 1D). Moreover, removal of the catheter didn't require any suture. She was followed up at our outpatient clinic for rib fractures after two months and no recurrent pneumothorax was found.

\section{Discussion}

The treatment of a small traumatic pneumothorax in the absence of respiratory manifestations has been controversial. However, if the patient has comorbid diseases which require surgery, in the face of positive pressure ventilation or general anesthesia, simple interventions such as percutaneous tube thoracostomy might be important. These procedures may be associated with significant morbidity when managed by personnel without appropriate training in trauma care. (13) In this situation, the catheter-based approach may be an alternative choice for chest tube or pigtail drainage.

Modified central venous catheters for pneumothorax have been reported and these authors considered the procedure to be effective, safe, easy to perform, portable, reliable and less painful. $(10,11)$ Anterior axillary line, 3rd or 4th intercostal space was chosen for most patients and the midclavicular line, 3rd intercostal space was used in obese patients. After central venous cathe- 
ter insertion, the catheter was fixed to the skin at $15-20 \mathrm{~cm}$. We followed the rules and the procedure was performed smoothly. These authors also suggested creating extra drainage holes in the central venous catheters to decrease the failure rate. However, we didn't cut extra holes due to the possibility of the tube breaking in the pleural space, and our patient had a good prognosis without any complications.

Our experience with this technique is encouraging. The placement of a central venous catheter is almost painless after administering local analgesia, and no intravenous sedation or analgesia is required. Moreover, it is less discomfor- ting when the catheter is indwelling. The patient may only feel slight discomfort when the catheter is inserted into the pleural space. Negative pressure can be applied via the appropriate connection of catheter to the chest bottle. It functions effectively to drain the pneumothorax. Finally, removal of the catheter doesn't require any suturing.

However, the technique has some limitations. First, our patient only had a simple pneumothorax. If a hemothorax presented, the blood might occlude the catheter and the drainage system may lose function. Second, due to the less invasive procedure, unlike traditional thoracotomy, we couldn't use our finger to dissect the pleural space and the sharp needle inserted during the procedure might have injured the lung without noticing. Therefore, slow insertion into the pleural space is indicated. Moreover, failure rate and the possibility of breaking the tube inside the pleural space may need further evaluation and be further determined.

In conclusion, a central venous catheter may be used to drain small, delayed traumatic pneumothoraxes as an alternative to chest tube or pigtail drainage, especially when positive pressure ventilation is indicated. It is effective and safe, easy to perform, and is less painful.

\section{REFERENCES}

1. Shorr RM, Crittenden M, Indeck M, Hartunian SL, Rodriguez A. Blunt thoracic trauma. Analysis of 515 patients. Ann Surg Aug 1987;206(2):200-5

2. Zinck SE, Primack SL. Radiographic and CT findings in blunt chest trauma. J Thorac Imaging Apr 2000;15(2):87-96.

3. Tekinbas C, Eroglu A, Kurkcuoglu IC, Turkyilmaz A, Yekeler E, Karaoglanoglu N. ŠChest trauma: analysis of 592 casesĆ. Ulus Travma Acil Cerrahi Derg Oct 2003;9(4):275-80.

4. Farooq U, Raza W, Zia N, Hanif M, Khan MM. Classification and management of chest trauma. J Coll Physicians Surg Pak Feb 2006;16(2):101-3.

5. Ball CG, Kirkpatrick AW, Laupland KB, Fox DI, Nicolaou S, Anderson IB, et al. Incidence, risk factors, and outcomes for occult pneumothoraces in victims of major trauma. J Trauma Oct 2005;59(4):917-24.

6. Lu MS, Huang YK, Liu YH, Liu HP, Kao CL. Delayed pneumothorax complicating minor rib fracture after chest trauma. Am J Emerg Med Jun 2008;26(5):551-4.

7. Misthos P, Kakaris S, Sepsas E, Athanassiadi K, Skottis I. A prospective analysis of occult pneumothorax, delayed pneumothorax and delayed hemothorax after minor blunt thoracic trauma. Eur J Cardiothorac Surg May 2004;25(5):859-64.

8. Wakai A, O'Sullivan RG, McCabe G. Simple aspiration versus intercostal tube drainage for primary spontaneous pneumothorax in adults. Cochrane Database Syst Rev 2007(1):CD004479.

9. Zehtabchi S, Rios CL. Management of emergency department patients with primary spontaneous pneumothorax: needle aspiration or tube thoracostomy? Ann Emerg Med Jan 2008;51(1):91-101.

10. Ishibashi H, Ohta S, Hirose M. Modified central venous catheter for pneumothorax. Gen Thorac Cardiovasc Surg Jun 2008;56(6):309-10.

11. Marshall MB. Modified central line for pneumothorax. Ann Thorac Surg Oct 2006;82(4):1543-4.

12. Seldinger SI. Catheter replacement of the needle in percutaneous arteriography; a new technique. Acta radiol May 1953;39(5):368-76.

13. Deneuville M. Morbidity of percutaneous tube thoracostomy in trauma patients. Eur J Cardiothorac Surg Nov 2002;22(5):673-8. 